Rev Inv Vet Perú 2017; 28(1): 01-12

http://dx.doi.org/10.15381/rivep.v28i1.12940

REVISIÓN DE LITERATURA

\title{
Fundamentos y Consideraciones de la Patología Endometrial Canina
}

\author{
Fundamentals and Considerations of the Canine Endometrial Pathology
}

Alfonso Sánchez Riquelme ${ }^{1,2}$, Francisco Arias Ruiz ${ }^{1}$

\section{Resumen}

\begin{abstract}
El estudio de las patologías reproductivas es de importancia para el adecuado abordaje clínico y médico. El objetivo del presente artículo fue realizar una investigación bibliográfica sobre los fundamentos y consideraciones de la patología endometrial canina, con énfasis en la hiperplasia endometrial quística y la piometra, buscando sostener o refutar el concepto arraigado o dogma, acerca de la condicionalidad existente entre ellas, el cual ha sido denominado complejo hiperplasia endometrial quística-piometra. De acuerdo a la evidencia analizada, se puede señalar que en la perra existen dos tipos de lesiones uterinas comunes que pueden ser de presentación independiente: alteraciones del crecimiento (hiperplasia) y alteraciones inflamatorias/infecciosas (endometritis-piometra). En la etiopatogenia de ambas entidades, el rol de la progesterona, en tanto generadora de cambios celulares y moleculares en el epitelio uterino, resulta indiscutible.
\end{abstract}

Palabras clave: canino, endometrio, piometra, hiperplasia endometrial quística

\section{Abstract}

The study of reproductive diseases is of great importance for clinical and medical approach. The aim of this article was to conduct a literature search on the grounds and considerations of canine endometrial pathology, with emphasis on endometrial cystic hyperplasia and pyometra, seeking support or refute the established concept or dogma, about the existing conditionality between them, which has been called cystic endometrial hyperplasia pyometra complex. According to the evidence, it is noted that there are two

\footnotetext{
${ }^{1}$ Escuela de Medicina Veterinaria, Facultad de Medicina Veterinaria y Agronomía, Universidad de Las Américas, Viña del Mar, Chile

${ }^{2}$ E-mail: profesanchez@gmail.com
}

Recibido: 22 de junio de 2016

Aceptado para publicación: 17 de octubre de 2016 
types of common uterine lesions in the bitch, that may occur independently: changes in growth pattern (hyperplasia) and inflammatory/infectious disorders (endometritispyometra). In the pathogenesis of both entities the role of progesterone, while generating cellular and molecular changes in the uterine epithelium, is indisputable.

Key words: canine, endometrium, pyometra, cystic endometrial hyperplasia

\section{INTRODUCCIÓN}

En la clínica de animales pequeños, la patología uterina más diagnosticada en caninos y, por consecuencia la más reconocida, es la piometra. Esta entidad se describe como una enfermedad posestral aguda o crónica, de hembras adultas enteras, que se caracteriza por acumulación de pus en el útero y signos reproductivos, sistémicos, hematológicos e imagenológicos variables (Feldman y Nelson, 1996; Tello et al., 1996; Johnston et al., 2001; Faldyna et al., 2001; Root Kustritz y Barber, 2003; Hagman, 2012; Haas et al., 2016).

La piometra también ha sido denominada piometritis, complejo piometra, endometritis catarral, endometritis purulenta, endometritis quística crónica, endometritis purulenta crónica y complejo hiperplasia endometrial quística-piometra, siendo esta última denominación la más utilizada (Feldman y Nelson, 1996; Harvey, 1998; Noakes et al., 2001; Johnston et al., 2001; Root Kustritz y Barber, 2003; Fontbonne, 2010; Kempisty et al., 2013).

Cabe destacar que el concepto de complejo hiperplasia endometrial-piometra (HEQP) de la perra fue introducido hace casi seis décadas a partir del estudio y la descripción de cuatro categorías de patología uterina, en las cuales el denominador común era la hiperplasia endometrial, un trastorno proliferativo progresivo asociado a la exposición crónica del revestimiento uterino a los esteroides ováricos, el cual puede generar quistes endometriales en su etapa más avan- zada (Dow, 1957, 1958, 1959). Si bien esta nomenclatura ha sido aceptada por diversos autores a través del tiempo (Feldman y Nelson, 1996; Harvey, 1998; Noakes et al., 2001; Johnston et al., 2001; Root Kustritz y Barber, 2003; Arora et al., 2016; Fontbonne, 2010; Kempisty et al., 2013), también ha sido señalada como un dogma de la patología reproductiva canina (Verstegen et al., 2008), pese a la evidencia aportada a inicios del siglo XXI por estudios que estarían indicando que la hiperplasia endometrial quística y la piometra serían entidades con desarrollos independientes (De Bosschere et al., 2001, 2002b).

Resulta llamativo observar que los mecanismos precisos de la patogénesis de la hiperplasia endometrial quística y su relación o posible relación con la presentación de piometra en la perra aún no son del todo bien comprendidos (Kida et al., 2010; Kempisty et. al., 2013, Enginler et al., 2014). Empero, en tiempos recientes, la incorporación de técnicas como biopsia uterina, inmunocitoquímica y análisis computarizado de imagen, así como también el mayor conocimiento de la función de los receptores uterinos a hormonas esteroidales, han permitido una nueva aproximación a la comprensión de la patología endometrial canina (Groppetti et al., 2010; Christensen et al., 2012; Schlafer, 2012).

El propósito de la presente revisión bibliográfica fue contribuir al conocimiento de la patología endometrial canina, entregando fundamentos y consideraciones sobre la relación o posible relación entre la hiperplasia endometrial quística y la piometra. 


\section{Fisiología Reproductiva de la Perra}

Entre los animales domésticos, la perra (Canis lupus familiaris) posee una biología reproductiva excepcional, clasificándosela como hembra monoéstrica típica, polítoca, no estacional y ovuladora espontánea (Concannon, 2011), con un intervalo interestral (IIE) entre 4 y 12 meses (Sánchez, 1999).

El ciclo reproductivo de la perra se caracteriza por un prolongado periodo de anestro, durante el cual la hembra no evidencia signos de actividad ovárica. A este periodo le sigue la fase del proestro, con una duración promedio de nueve días, que se caracteriza por una importante actividad folicular y secreción de $17 \beta$-estradiol, con la aparición de signos clínicos tales como edema vulvar, descarga vaginal sanguinolenta y atracción del macho. Endocrinológicamente, el proestro finaliza con la aparición del pico de hormona luteinizante (LH) (Concannon, 2011). En la fase siguiente, el estro, aparece la receptividad sexual y una marcada queratinización del epitelio vaginal, con una duración promedio de nueve días. Durante el primer tercio del estro suele ocurrir el pico de LH y la ovulación con el consecuente desarrollo de los cuerpos hemorrágicos y cuerpos lúteos (Groppetti et al., 2015). Una vez finalizada la receptividad sexual y que el epitelio vaginal disminuye significativamente el nivel de queratinización celular, se señala que ha comenzado el metaestro o diestro (fase lútea), durante el cual, los niveles plasmáticos de progesterona $\left(\mathrm{P}_{4}\right)$ se mantienen elevados $(>2 \mathrm{ng} / \mathrm{ml})$ por alrededor de 60 a 90 días (Concannon, 2011).

En la perra, a través del ciclo estral, el revestimiento uterino experimenta una serie de modificaciones macroscópicas, microscópicas y moleculares, asociados principalmente a la influencia de estrógenos y $\mathrm{P}_{4}$ (Fernandes et al., 1989; De Bosschere et al., 2002a; Schlafer, 2012). En la fase estrogénica ocurre proliferación celular e incremento de la vascularización, mientras que en la fase progestacional (diestro), el endometrio presenta dos etapas principales de crecimiento y otra de diferenciación (Barrau et al., 1975). Luego, desde el diestro medio hasta el anestro temprano, es característica la presencia de eventos degenerativos y regenerativos del endometrio (Groppetti et al., 2010).

Durante el diestro, por efecto de la $\mathrm{P}_{4}$, el endometrio tiene sus glándulas plenamente desarrolladas y activas. Esta condición predispone a la perra al desarrollo de la hiperplasia endometrial quística y la piometra, patologías características de esta etapa del ciclo estral (Gobello, 2011).

\section{Fundamentos y Consideraciones de la Patología Endometrial Canina}

\section{Hiperplasia endometrial quística}

La hiperplasia endometrial quística (HEQ) de la perra es considerada como un trastorno reproductivo subclínico que puede o no afectar la fertilidad y se le ha asociado con una respuesta anormal del endometrio a los esteroides ováricos. Se caracteriza por engrosamiento del endometrio, atribuido al aumento en número y tamaño de las células epiteliales y de las glándulas endometriales (Schlafer, 2012).

La prolongada estimulación hormonal durante la fase diestral del ciclo sexual de la perra condicionaría un incremento en el número y la actividad de las células secretoras, con la consiguiente acumulación de exudados en el útero (Chen et al., 2001). Además, esta hiperplasia e hipertrofia glandular con hipersecreción de las glándulas endometriales facilitaría la formación de quistes con líquido estéril en el útero (Verstegen et al., 2008; Schlafer, 2012). Varios autores estiman que la HEQ es un estado previo y necesario para la presentación de piometra en la perra (Feldman y Nelson, 1996; Harvey, 1998; Johnston et al., 2001; Root Kustritz y Barber, 2003; Fontbonne, 2010). 
Desde hace décadas, el complejo HEQ$\mathrm{P}$ ha sido asociado con desórdenes hormonales, tales como quistes ováricos, hiperestrogenismo y ovarios poliquísticos (Dow, 1958). En este sentido, existe concordancia en que la administración de progestágenos o estrógenos resulta en una mayor incidencia de HEQ-P (Feldman y Nelson, 1996; Johnston et al., 2001; Noakes et al., 2001; Root Kustritz y Barber, 2003; Kida et al., 2006; Smith, 2006; Pretzer, 2008; Kempisty et al., 2013).

No obstante, el complejo HEQ-P puede afectar a hembras a partir del primer ciclo, pero el riesgo de presentación del complejo HEQ-P es mayor en perras nulíparas mayores de 4 años (Verstegen et al., 2008). Asimismo, Niskanen y Thrusfield (1998) señalan que el complejo HEQ-P es relativamente común en perras enteras entre 8 y 9 años de edad, lo cual tendría una relación con el incremento de la prevalencia de HEQ en perras de mayor edad (Moxon et al., 2016). Por otro lado, algunos autores describen cierto grado de protección dado por la pluriparidad de las hembras ante el eventual desarrollo de la enfermedad, pero esto aún no es concluyente (Hagman et al., 2011).

Un aspecto característico del complejo HEQ-P es que ocurre principalmente durante la fase lútea del ciclo sexual, clasificándosela de este modo como una patología diestral; situación que ha motivado un especial interés por el papel que desempeña la $\mathrm{P}_{4}$ en la patogénesis de dicha enfermedad (Nomura, 1985; Harvey, 1998). Por lo pronto, no se han descrito diferencias significativas en los niveles plasmáticos de $\mathrm{P}_{4}$ comparando perras sanas y perras con el complejo HEQ-P (Hadley, 1975; Nomura, 1985; Gultiken et al., 2016), así como tampoco alguna correlación entre concentración plasmática de $\mathrm{P}_{4}$ e incidencia de piometra (Kang et al., 1995, citado por Johnston et al., 2001).
No obstante la ausencia de relación entre los niveles plasmáticos de $\mathrm{P}_{4} \mathrm{y}$ la presentación de piometra, llama la atención que perras en fase lútea temprana o media, a las cuales se les indujo irritación endometrial, experimentaron una reacción caracterizada por proliferación y dilatación glandular, similar a la HEQ; mientras que aquellas hembras que al momento de inducir la irritación endometrial se encontraban en fase lútea tardía no presentaron dicha reacción (Nomura, 1997; Nomura y Nishida, 1998). Estos antecedentes indicarían que para el desarrollo de HEQ se requiere de niveles plasmáticos de $\mathrm{P}_{4}$ asociados a la presencia de cuerpos lúteos funcionales y que factores asociados con trauma o irritación endometrial actuarían sinérgicamente con el estímulo progestacional.

\section{Endometritis}

La inflamación endometrial se encuentra estrechamente relacionada con la producción de factores de crecimiento. En forma similar, la prolongada secreción de $\mathrm{P}_{4}$ en cada ciclo estral puede ocasionar el incremento en la secreción de hormona del crecimiento, con el consiguiente aumento del factor insulínico de crecimiento tipo I (IGF-I), factor que estimula la proliferación endometrial, proponiéndose de esta forma un rol de IGF-I en la patogénesis del complejo HEQ-P en la perra (De Cock et al., 2002). Así también, se ha propuesto que el factor de crecimiento transformante $\alpha$ (TGF- $\alpha$ ) producido por las células inflamatorias, en presencia de una baja expresión y diferenciación local del factor epidermal de crecimiento (EGF), puede condicionar un patrón de proliferación aberrante en las glándulas endometriales y condicionar la presentación del complejo HEQ-P (Kida et al., 2010).

Una estrategia para el estudio de la patología endometrial en la perra ha sido la estimulación mecánica del endometrio, mediante la inserción de cuerpos extraños en el lumen del útero (Nomura, 1997; Nomura y 
Makino, 1997; Nomura y Nishida, 1998; Dhaliwal et al., 2001; De Bosschere et al., 2002c). Nomura (1997) propuso llamar deciduoma canino a aquella respuesta endometrial ocurrida en diestro ante un estímulo artificial inespecífico, ya que se caracteriza por proliferación, dilatación glandular e hiperplasia endometrial quística, respuesta semejante a la reacción decidual del endometrio en roedores. Además, se ha descrito que los cambios uterinos que ocurren cuando se induce el deciduoma canino son similares a los cambios que experimenta el útero en casos de piometra espontánea (Nomura y Funahashi, 1999).

Schlafer y Gifford (2008) han definido una forma particular de cambios quísticos en el endometrio canino, conocida como hiperplasia endometrial pseudo-placentacional (HEP), destacando que existe una marcada proliferación endometrial con patrones difusos o segmentarios, semejantes a lo observado en las regiones de placentación zonal. La presencia de vellosidades en la superficie endometrial, con formación de pliegues que pueden fusionarse, explicaría la formación de quistes. Además, los cambios proliferativos implicarían la posibilidad de ruptura vascular con extravasación, de manera similar a lo observado en el deciduoma de la placentación endoteliocorial zonal canina (Nomura y Funahashi, 1999), condición que ha sido propuesta como posible causa de hematometra en perras (Sánchez, 2015). La HEP sería una condición predisponente para el desarrollo de endometritis y la consiguiente piometra (Schlafer, 2012).

\section{Rol de los receptores hormonales}

También ha sido sugerido que el trauma endometrial predispondría al desarrollo de HEQ, a través de un mecanismo de modificación de receptores a hormonas esteroidales, de una manera similar a como sería por bacterias patógenas. Este mecanismo incluiría factores que conducirían a un incremento de la sensibilidad del tejido uterino a la influencia de hormonas esteroidales endógenas
(Dhaliwal et al., 2001). En este sentido, cabe agregar que, desde una perspectiva histológica, la reacción endometrial inducida por estímulo mecánico es similar a la HEQ espontánea (Nomura, 1997); sin embargo, la expresión de receptores a hormonas sexuales en dichas condiciones no es similar, lo cual agrega una dificultad más a la comprensión de dicha patología (De Bosschere et al., 2002c).

El rol de los receptores endometriales a esteroides sexuales ha recibido especial atención, postulándose que cambios en la expresión y la distribución de dichos receptores en respuesta, tanto a hormonas endógenas como exógenas, podrían estar involucrados en la patogénesis de la HEQ (Fernandes et al., 1989; De Cock et al., 1997; Dhaliwal et al., 1999; Vermeirsch et al., 1999, 2000; De Bosschere et al., 2002a,b).

De Cock et al. (1997), estudiando úteros caninos con HEQ, describen una alteración en la regulación de la población de receptores de estrógenos durante la fase lútea, caracterizada por una expresión prolongada de los mismos, lo cual podría asociarse a una proliferación anormal de glándulas endometriales. Cabe agregar que esto adquiere particular importancia, si se considera que durante el diestro de la perra existe influencia estrogénica, la que se asociaría a la secreción luteal de 17 $\beta$-estradiol (Onclin et al., 2002; Papa y Hoffmann, 2011).

Comparando la densidad uterina de receptores de estrógenos y de $\mathrm{P}_{4}$, se observaron diferencias entre perras con HEQmucometra y perras con endometritispiometra, sugiriendo que cambios en la expresión de estos receptores estaría involucrada en la patogénesis de uno u otra forma de la enfermedad endometrial de la perra (De Bosschere et al., 2002b). Estos autores destacan que dichos cambios no serían la causa primaria del desarrollo de HEQ y proponen una vía hormonal para la presentación de HEQ-mucometra en perras tratadas con progestágenos, agregando que la 
infección bacteriana sería el factor desencadenante del complejo endometritis-piometra.

Chu et al. (2001) evaluaron el efecto del estado hormonal de la hembra sobre la presentación de HEQ inducida. Para ello utilizaron perras ovariectomizadas, reportando una marcada dilatación de las glándulas endometriales en hembras tratadas con estradiol y luego con progesterona. Los resultados de este estudio indican que el estradiol sensibilizaría al endometrio, potenciando la acción de la $\mathrm{P}_{4}$ para el desarrollo de HEQ. Efecto que podría estar mediado por la inducción de receptores endometriales de $\mathrm{P}_{4}$ (Dhaliwal et al., 1999).

Un aspecto novedoso en el estudio de la fisiopatología del complejo HEQ-P es el rol de la síntesis de $\mathrm{P}_{4}$ endometrial. Gultiken et al. (2016), comparando la expresión de la enzima $3 \beta$-hidroxiesteroide dehidrogensa ( $3 \beta$ HSD) en tejido uterino de perras en diestro versus tejido uterino de perras con HEQ-P, describen una expresión significativamente superior en este último grupo, sugiriendo un efecto intracrino de la $\mathrm{P}_{4}$ en la etiología de esta patología.

\section{Mucometra y piometra}

De Bosschere et al. (2001), analizando genitales de perras sanas y con antecedentes de patología uterina, destacaron que en hembras clínicamente sanas solo se observaron signos moderados a severos de HEQ, mientras que en hembras con signología clínica se presentó reacción inflamatoria del endometrio. De esta forma, proponen que el complejo HEQ-P de la perra podría ser dividido en dos entidades: a) HEQ-mucometra y b) endometritis-piometra. Además, existiría evidencia histológica e inmunocitoquímica de que la HEQ no es un requisito para el desarrollo de piometra, como sí lo sería la presencia de endometritis (De Bosschere et al., 2001, 2002b).
Es así que se podría planear que el complejo endometritis-piometra sería una enfermedad clínica, mientras que el complejo HEQmucometra correspondería más bien a un hallazgo incidental durante la ovariohisterectomía, ya que esta condición raramente arroja signos clínicos (Johnston et al., 2001). Por otra parte, ya que la HEQ puede llevar a una acumulación de fluido estéril en el útero y que, dependiendo del grado de hidratación de la mucina, la resultante puede ser hidrometra o mucometra, se ha propuesto que la mucometra correspondería a un estado avanzado de HEQ, caracterizado por atrofia de la pared uterina y presencia de secreción mucofilamentosa, lo que se traduciría en alteraciones de la fertilidad (Verstegen et al., 2008).

\section{Rol de las bacterias}

Un aspecto determinante en la patogenia del complejo endometritis-piometra en la perra es la presencia de bacterias en el útero (Nomura, 1985; De Bosschere et al., 2001; Noakes et al., 2001; De Bosschere et al., 2002c). Así también, se ha destacado la ausencia de bacterias en el útero de perras que solo presentaban HEQ (Chen et al., 2001; Dhaliwal et al., 2001). La inoculación de $E$. coli directamente en el lumen uterino de perras sanas en diestro produjo cambios histológicos característicos del deciduoma canino, acompañados de severa inflamación y posterior presentación de piometra (Nomura y Funahashi, 1999).

La endometritis bacteriana, en su fase inicial, se caracteriza por una reacción celular neutrofilica en la superficie del endometrio, acompañada de congestión vascular y edema subyacente al epitelio luminal. Los neutrófilos también se encuentran marginados a lo largo de las paredes internas de los capilares del estrato compacto. El epitelio luminal a menudo es irregular y en casos graves puede estar ulcerado. En la endometritis crónica, la composición del infiltrado inflama- 
torio cambia e incluye presencia de linfocitos, células plasmáticas y macrófagos, distribuyéndose hacia las capas más profundas del estroma endometrial (McEntee, 1990; Schlafer, 2012).

Dado que el cérvix canino se encuentra abierto durante la fase folicular del ciclo estral (Silva et al., 1995), las bacterias llegarían al útero vía ascendente desde la vagina (Johnston et al., 2001). Los signos clínicos de la enfermedad, comúnmente observados entre las 6 y 8 semanas del estro (Harvey, 1998), se asociarían directamente con la producción de toxinas e indirectamente con la liberación de mediadores de la inflamación (Faldyna et al., 2001; Haas et al., 2016). Los microorga-nismos aislados con mayor frecuencia a partir de úteros con piometra son E. coli, Streptococcus sp, Staphilococcus sp, Pseudomonas sp y Proteus sp, correspondiendo el 70 a $90 \%$ de los casos a E. coli (Dhaliwal et al., 1998; Hagman y Kühn, 2002; Agostinho et al., 2014). El efecto de la $\mathrm{P}_{4}$ sobre las células endometriales tendría la capacidad de sensibilizar al epitelio para la infección por E. coli, particularmente a cepas reconocidas como urogenitales, las cuales poseen factores de virulencia especializados que favorecen la infección (Chen et al., 2003).

Se ha descrito que en el endometrio canino ocurren cambios durante el diestro en la expresión de azúcares de superficie que permiten la infección bacteriana, influyendo en la presentación de piometra (Yasunaga et al., 2013). Por otra parte, Tsumagari et al. (2005) señalan que en perras a las cuales se les indujo piometra por inoculación intrauterina de E. coli, el periodo de mayor susceptibilidad para desarrollar la patología es el diestro temprano, bajo la influencia de altos niveles de $\mathrm{P}_{4}$ plasmática. Esto guardaría relación con lo descrito por Gabriel et al. (2016), quienes observaron que en úteros con piometra existe una alta expresión del receptor funcional de lipoproteína de alta densidad (SR-B1) respecto de endometrios sanos en fase de diestro, sugiriéndose una posible interacción entre las bacterias y el SR-B1 que favorecería la adhesión y el paso de bacterias a través de la barrera epitelial.

Cabe considerar que la respuesta uterina a la presencia de bacterias se basa en gran medida en la inmunidad innata, y que los patrones moleculares asociados a patógenos (PAMP) sintetizados por los microorganismos, tales como lipopolisacáridos (LPS) para las bacterias Gram negativas, son reconocidos por los receptores tipo Toll (TLRs) de las células endometriales, iniciando una respuesta inflamatoria no específica con reclutamiento de células del sistema inmune, incluyendo los neutrófilos polimorfonucleares (PMN) (Horne et al., 2008).

Las endotoxinas de E. coli pueden producir algunos de los signos clínicos característicos de piometra, tales como letargia, depresión, anorexia, poliuria, polidipsia, vómito y fiebre (Faldyna et al., 2001; De Bosschere et al., 2002c). Las endotoxinas, al ser reconocidas por los TLR desencadenan el inicio de una cascada de señales que provocan una disfunción en los PMN y macrófagos locales, los que comienzan la liberación masiva de citoquinas, responsables de la inflamación y de varios de los signos sistémicos que presentan las hembras con piometra. Estos signos están asociados a mediadores inflamatorios secundarios, como serían los radicales libres del oxígeno (ROS), óxido nitroso y prostaglandinas (Hagman et al., 2006; Dabrowski et al., 2007; Chotimanukul y Sirivaidyapong, 2011).

Cabe destacar que uno de los aspectos principales, en cuanto a la presentación de signos clínicos en hembras con piometra, es el grado de abertura del cérvix durante el desarrollo de la patología, donde se reconocen dos presentaciones: piometra a cérvix cerrado (distensión y acumulación de pus) y piometra a cérvix abierto (descarga vulvar), siendo la primera la más grave, ya que implica mayor compromiso sistémico y riesgo de muerte (Pretzer, 2008; Kempisty et al., 2013). 
La regulación del grado de abertura cervical durante el desarrollo de la piometra canina es una condición no bien comprendida; no obstante, se ha postulado que el incremento en el número de neutrófilos en la cérvix y el aumento de la actividad de la colagenasa cervical podrían estar relacionados con la expresión local de interleuquina 8 (IL-8), con la consiguiente degradación del colágeno y la remodelación del tejido conectivo del cuello uterino, permitiendo su abertura (Tamada et al., 2012). En este sentido, cabe destacar que se han descrito niveles plasmáticos de IL-8 significativamente superiores en perras con piometra moderada respecto de hembras con cuadros sistémicos severos (Haas et al., 2016). Así también, ha sido sugerido que la expresión de genes de ciclooxigensa 2 (COX2) y prostaglandina E sintetasa (PGES) durante la inflamación endometrial podrían estar implicados en la regulación del grado de abertura cervical en perras con piometra (Tamada el al., 2016).

Como conclusión, se puede señalar que no obstante existir abundante investigación en torno a la enfermedad endometrial canina, no existe plena claridad en cuanto a la etiopatogenia de un grupo de patologías concomitantes, como sería la relación sistemáticamente propuesta entre hiperplasia endometrial quística y piometra, utilizándose un lenguaje técnico que tiende a uniformar dichas patologías en la forma de un complejo. No obstante, para el caso de ambas entidades señaladas, el rol de la progesterona como generadora de cambios celulares y moleculares en el epitelio uterino resulta indiscutible. Bajo esta óptica, es importante continuar los estudios para dilucidar lo que, a la luz de los antecedentes, pareciera ser un dogma de la patología.

\section{Literatura Citada}

1. Agostinho J, de Souza A, SchockenIturrino R, Beraldo L, Borges C, Avila F, Marin J. 2014. Escherichia coli strains isolated from the uteri horn, mouth, and rectum of bitches suffering from pyometra: virulence factors, antimicrobial susceptibilities, and clonal relationships among strains. Int $\mathrm{J}$ Microbiol 979584. 8 p. doi: $10.1155 / 2014 /$ 979584

2. Arora N, Sandford J, Browning G, Sandy J, Wright P. 2006. A model for cystic endometrial hyperplasia/pyometra complex in the bitch. Theriogenology 66 : 1530-1536.

3. Barrau M, Abel J, Verhage H, Tietz W. 1975. Development of the endometrium during the estrous cycle in the bitch. Am J Anat 142: 47-65. doi: 10.1002/aja.1001420105

4. Chen Y, Wright P, Lee C. 2001. A model for the study of cystic endometrial hyperplasia in bitches. J Reprod Fert Suppl 57: 407-414.

5. Chen Y, Wright P, Lee C, Browning G. 2003. Uropathogenic virulence factors in isolates of Escherichia coli from clinical cases of canine pyometra and feces of healthy bitches. Vet Microbiol 94: 57-69.

6. Chotimanukul S, Sirivaidyapong S. 2011. Differential expression of Toll-like receptor 4 (TLR4) in healthy and infected canine endometrium. Theriogenology 76: 1152-1161. doi: 10.1016/ j.theriogenology.2011.05.024

7. Christensen B, Schlafer D, Agnew D, Wang C, Kozlowski C, Asa C. 2012. Diagnostic value of transcervical endometrial biopsies in domestic dogs compared with full-thickness uterine sections. Reprod Dom Anim 47 (Suppl 6): 342-346. doi: 10.1111/rda.12109

8. Chu P, Lee C, Moore P, Wright, P. 2001. Oestrogen and progestagen treated ovariectomized bitches: a model for the study of uterine function. J Reprod Fert Suppl 57: 45-54.

9. Concannon P. 2011. Reproductive cycles of the domestic bitch. Anim Reprod Sci 124: 200-210. doi: 10.1016/ j.anireprosci.2010.08.028 
10. Dabrowski R, Wawron W, Kostro K. 2007. Changes in CRP, SAA and haptoglobin produced in response to ovariohysterectomy in healthy bitches and those with pyometra. Theriogenology 67: 321-327. doi: 10.1016/j.theriogenology.2006.07.019

11. Dhaliwal G, Wray C, Noakes D. 1998. Uterine bacterial flora and uterine lesions in bitches with cystic endometrial hyperplasia (pyometra). Vet Rec 143: 659-661. doi: 10.1136/vr.143.24.659

12. Dhaliwal G, England G, Noakes D. 1999. The influence of exogenous steroids hormones on steroid receptors, uterine histological structure and the bacterial flora of the normal bitch. Anim Reprod Sci 56: 259-277.

13. Dhaliwal G, England G, Noakes D. 2001. The effects of endometrial scarification on uterine steroids receptors, bacterial flora and histological structure in the bitch. Anim Reprod Sci 69: 239-249. doi: 10.1016/S0378-4320(01)00183-X

14. De Bosschere H, Ducatelle R, Vermeirsch H, Van Den Broek W, Coryn M. 2001. Cystic endometrial hyperplasia - pyometra complex in the bitch: should the two entities be disconnected? Theriogenology 55: 15091519. doi: 10.1016/S0093-691X(01) 00498-8

15. De Bosschere H, Ducatelle R, Tshamala M, Coryn M. $2002 a$. Changes in sex hormone during administration of progesterone to prevent estrus in bitch. Theriogenology 58: 12091217. doi: 10.1016/S0093-691X(02) 00951-2

16. De Bosschere H, Ducatelle R, Vermeirsch H, Simoens P, Coryn M. 2002b. Estrogen- $\alpha$ and progesterone receptor expression in cystic endometrial hyperplasia and pyometra in the bitch. Anim Reprod Sci 70: 251-259. doi: 10.1016/S0378-4320(02)00013-1

17. De Bosschere H, Ducatelle R, Tshamala M. 2002c. Is mechanically induced cystic endometrial hyperplasia (CEH) a suitable model for study of spontaneously occurring CEH in the uterus of the bitch? Reprod Dom Anim 37: 152-157. doi: 10.1046/j.14390531.2002.00358.x

18. De Cock H, Ducatelle R, Logghe J. 1997. Immunohistochemical localization of estrogen receptors in the normal canine female genital tract. Domest Anim Endocrinol 14: 133-147. doi : 10.1016/ s0739-7240(97)00001-5

19. De Cock H, Ducatelle R, Tilmant K, De Schepper J. 2002. Possible role for insuline-like factor-I in the pathogenesis of cystic endometrial hyperplasia pyometra complex in the bitch. Theriogenology 57: 2271-2287. doi: 10.1016/S0093-691X(02)00856-7

20. Dow C. 1957. The cystic hyperplasiapyometra complex in the bitch. Vet Rec 69: 1404-1414.

21. Dow C. 1958. The cystic hyperplasiapyometra complex in the bitch. Vet Rec 70: 1102-1108.

22. Dow C. 1959. Experimental reproduction of the cystic hyperplasia pyometra complex in the bitch. J Pathol 78: 267278. doi: 10.1002/path.1700780129

23. Enginler S, Ates A, Diren Sig-rci B, Sonta] B, Sönmez K, Karacam E, Ekicil H, et al. 2014. Measurement of C-reactive protein and prostaglandin $\mathrm{F}_{2 a}$ metabolite concentrations in differentiation of canine pyometra and cystic endometrial hyperplasia/ mucometra. Reprod Dom Anim 49: 641647. doi: $10.1111 /$ rda. 12340

24. Faldyna M, Laznicka A, Toman M. 2001. Immunosuppression in bitches with pyometra. J Small Anim Pract 42: 5-10. doi: 10.1111/j.1748-5827.2001. tb01976.x

25. Feldman E, Nelson R. 1996. Canine and feline endocrinology and reproduction. Philadelphia, USA: WB Saunders. $785 \mathrm{p}$.

26. Fernandes P, Bowen $R$, Sawyer H, Nett T, Gorell T. 1989. Concentration of receptors for estradiol and proges-terone in canine endometrium during estrus and diestrus. Am J Vet Res 50: 64-67. 
27. Fontbonne A. 2010. Clinical approach to conditions of the non-pregnant and neutered bitch. In: England G, von Heimendahl A (eds). BSAVA Manual of canine and feline reproduction and neonatology. $2^{\text {nd }}$ ed. England: BSAVA. p 166-184.

28. Gabriel C, Becher-Deichsel A, Hlavaty J, Mair G, Walter I. 2016. The physiological expression of scavenger receptor SR-B1 in canine endometrial and placental epithelial cells and its potential involvement in pathogenesis of pyometra. Theriogenology 85: 15991609. doi: $10.1016 / \mathrm{j}$.theriogenology. 2016.01.021

29. Gobello C. 2011. Fisiología reproductiva aplicada canina. En: I Simposio Latinoamericano de Reproducción Animal. Chile.

30. Groppetti D, Pecile A, Arrighi, Di Giancamillo A, Cremonesi F. 2010. Endometrial cytology and computerized morphometric analysis of epihitelial nuclei: a usefull tool for reproductive diagnosis in the bitch. Theriogenology 73 : 927-941. doi: 10.1016/j.theriogenology. 2009.11.019

31. Groppetti D, Aralla M, Bronzo V, Bosi G, Pecile A, Arrighi S. 2015. Periovulatory time in the bitch: What's new to know? Comparison between ovarian histology and clinical features. Anim Reprod Sci 152: 108-116. doi: 10.1016/j.anireprosci.2014.11.008

32. Gultiken N, Yarim M, Yarim G, Gacar A, Mason J. 2016. Expression of $3 \beta$ hydroxysteroid dehydrogenase in ovarian and uterine tissue during diestrus and open cervix cystic endometrial hyperplasiapyometra in the bitch. Theriogenology 86 : 572-578. doi: 10.1016/j.theriogenology.2016.02.006

33. Haas M, Kaup F, Neumann S. 2016. Canine pyometra: a model for the analysis of serum CXCL8 in inflammation. J Vet Med Sci 78: 375-381. doi: 10.1292/jvms.15-0415
34. Hadley J. 1975. The development of cystic endometrial hyperplasia in the bitch following serial uterine biopsies. J Small Anim Pract 16: 249-257. doi: 10.1111/ j.1748-5827.1975.tb05741.x

35. Hagman R, Kuhn I. 2002. Escherichia coli strains isolated from the uterus and urinary bladder of bitches suffering from pyometra: comparison by restriction enzyme digestion and pulsedfield gel electrophoresis. Vet Microbiol 84: 143-153. doi: 10.1016/S03781135(01)00449-7

36. Hagman R, Kindahl H, Fransson B, Bergström A, Ström-Holst B, Lagerstedt A. 2006. Differentiation between pyometra and cystic endometrial hyperplasia/mucometra in bitches by prostaglandin F2 alpha metabolite analysis. Theriogenology 66: 198-206.

37. Hagman $R$, Lagerstedt $A$, Hedhammar A, Egenvall A. 2011. A breed-matched case-control study of potential risk-factors for canine pyometra. Theriogenology 75: 12511257. doi: $10.1016 / j$.theriogenology.2010.11.038

38. Hagman R. 2012. Clinical and molecular characteristics of pyometra in female dog. Reprod Dom Anim 47 (Suppl 6): 323-325. doi: 10.1111/ rda.12031

39. Harvey M. 1998. Condition of the nonpregnant female. In: Simpson G, England G, Harvey M (eds). BSAVA Manual of small animal reproduction and neonatology. England: BSAVA. p 35-51.

40. Horne A, Stock S, King A. 2008. Innate immunity and disorders of the female reproductive tract. Reproduction 135: 739-749. doi: 10.1530/REP-07-0564

41. Johnston S, Root Kustritz M, Olson P. 2001. Disorders of the canine uterus and uterine tubes (oviducts). In: Johnston S, Root Kustritz M, Olson P (eds). Canine and feline theriogenology. USA: WB Saunders. p 206-224. 
42. Kang B, Park I, Park N. 1995. Experimental production of canine pyometra by inoculation of Escherichia coli into the uterus. Korean J Vet Clin Med 124: 31-39.

43. Kempisty B, Bukowska D, Wozna M, Piotrowska H, Jackowska M, Zuraw $A$, et al. 2013. Endometritis and piometra in bitches: a review. Veterinarni Medicina 58: 289-297.

44. Kida K, Baba E, Torii R, Kawate N, Hatoya S, Wijewardana V, Sugiura K, et al. 2006. Lactoferrin expression in the canine uterus during the estrous cycle and with pyometra. Theriogenology, 66: 1325-1333.

45. Kida K, Maezono Y, Kawate N, Inaba T, Hatoya S, Hamada H. 2010. Epidermal growth factor, transforming growth factor-alpha, and epidermal growth factor receptor expression and localization in the canine endometrium during the estrous cycle and in bitches with pyometra. Theriogenology 73: 3647. doi: $10.1016 /$ j.theriogenology. 2009.08.002

46. Moxon R, Whiteside $H$, England G. 2016. Prevalence of ultrasounddetermined cystic endometrial hyperplasia and the relationship with age in dogs. Theriogenology 86: 976-80. doi: 10.1016/ j.theriogenology.2016.03.022

47. McEntee K. 1990. Reproductive pathology of domestic mammals. San Diego, CA, USA: Academic Press. p 171-176.

48. Niskanen M, Thrusfield M. 1998. Associations between age, parity, hormonal therapy and breed, and pyometra in Finnish dogs. Vet Rec 143: 493-498. doi: 10.1136/vr.143.18.493

49. Noakes D, Dhaliwal G, England G. 2001. Cystic endometrial hyperplasia/ pyometra in dogs: a review of the causes and pathogenesis. J Reprod Fert Suppl 57: 395-406.

50. Nomura K. 1985. Progesterone receptors in the canine endometrium affected with pyometra. J Jap Vet Med Ass, 38:174-176. doi: 10.12935/ jvma1951.38.174
51. Nomura K. 1997. Induction of canine deciduoma in some reproductive stages with the different conditions of corpora lutea. J Vet Med Sci 59: 185-190.

52. Nomura K, Makino T. 1997. Effect of ovariectomy in the early first half of the diestrus on induction or maintenance of canine deciduoma. J Vet Med Sci 59: 227-230.

53. Nomura K, Nishida A. 1998. Histological variations of canine deciduoma induced in non pregnant horn at different stages of unilateral pregnancy J Vet Med Sci 60: 623-626. doi: $10.1292 /$ jvms. 60.623

54. Nomura K, Funahashi H. 1999. Histological characteristics of canine deciduoma induced by intrauterine inoculation of $E$. coli suspension. J Vet Med Sci 61: 433-438.

55. Onclin K, Murphy B, Verstegen J. 2002. Comparison of estradiol, LH and FSH patterns in pregnant and nonpregnant beagle bitches. Theriogenology 57: 1957-1972. doi: 10.1016/S0093-691X(02)00644-1

56. Papa P, Hoffmann B. 2011. The corpus luteum of the dog: source and target of steroid hormones? Reprod Dom Anim 46: 750-756. doi: 10.1111/j.14390531.2010.01749.x

57. Pretzer S. 2008. Clinical presentation of canine pyometra and mucometra: a review. Theriogenology 70: 359-363. doi: 10.1016/j.theriogenology.2008.04.028

58. Root Kustritz M, Barber J. 2003. Uterine disorders. In: Root Kustritz M (ed). Small animal theriogenology. USA: Elsevier Science. p 367-394.

59. Sánchez A. 1999. Factores que afectan el intervalo interestral en la perra: una revisión. Rev CientífFCV-LUZ 9: 532-536.

60. Sánchez A. 2015. Hematometra e hiperplasia endometrial quística en una perra: descripción de un caso. Rev Inv Vet Perú 26: 146-151. doi: 10.15381/ rivep.v26i1.10918

61. Schlafer D, Gifford A. 2008. Cystic endometrial hyperplasia, pseudoplacentational endometrial hyperplasia, and 
other cystic conditions of the canine and feline uterus. Theriogenology 70: 349358. doi: $10.1016 / j$.theriogenology.2008.04.041

62. Schlafer D. 2012. Diseases of the canine uterus. Reprod Dom Anim 47 (Supp1 6): 318-322. doi: 10.1111/ rda.12064

63. Silva L, Onclin K, Verstegen J. 1995. Cervical opening in relation to progesterone and oestradiol during heat in beagle bitch. J Reprod Fert 104: 85-90.

64. Smith F. 2006. Canine pyometra. Theriogenology 66: 610-612. doi: 10.1016/j.theriogenology.2006.04.023

65. Tamada H, Kawata $N$, Kawate $N$, Inaba, T, Kida K, Hatoya S, Akune A, et al. 2012. Factors associated with patency of the uterine cervix in bitches with pyometra. Res Vet Sci 93: 12031210. doi: 10.1016/j.rvsc.2012.05.012

66. Tamada H, Adachi $N$, Kawate $N$, Inaba T, Hatoya S, Sawada T. 2016. Positive correlation between patency and mRNA levels for cyclooxygenase and prostaglandin E synthase in the uterine cervix of bitches with pyometra. J Vet Med Sci 78: 525-528. doi: 10.1292/ jvms. 15-0520

67. Tello L, Martín F, Valdés A, Albala A. 1996. Estudio comparativo de signos, ecográficos, radiográficos y postquirúrgicos en 50 perras con piometra. Arch Med Vet 28: 137-143.
68. Tsumagari S, Ishinazaka T, Kamata H, Ohba S, Tanaka S, Ishii M, Memon M. 2005. Induction of canine pyometra by inoculation of Escherichia coli into the uterus and its relationship to reproductive features. Anim Reprod Sci 87: 301-308. doi: 10.1016/j.anire-prosci.2004.11.006

69. Vermeirsch H, Simoens P, Lauwers H, Corny M. 1999. Immunohistochemical detection of estrogen receptors in the canine uterus and their relation to sex steroid hormone levels. Theriogenology 51: 729-743. doi: 10.1016/S0093691X(99)00022-9

70. Vermeirsch H, Simoens P, Hellemans L, Corny M, Lauwers H. 2000. Immunohistochemical detection of progesterone receptors in the canine uterus and their relation to sex steroid hormone levels. Theriogenology 53: 773-788. doi: 10.1016/S0093-691X(99)00273-3

71. Verstegen J, Dhaliwal G, VerstegenOnclin K. 2008. Mucometra, cystic endometrial hyperplasia, and piometra in the bitch: advances in treatment and assessment of future reproductive success. Theriogenology 70: 364-274. doi: 10.1016/ j.theriogeno-logy. 2008. 04.036

72. Yasunaga Y, Takeuchi T, Shimokawa T, Asano A, Nabeta M, Ohta Y. 2013. Sugar expression in the mucosae of the canine uterus and vagina during the oestrous cycle and with pyometra. Vet $\mathrm{J}$ 196: 116-118. doi: 10.1016/j.tvj1.2012. 08.008 